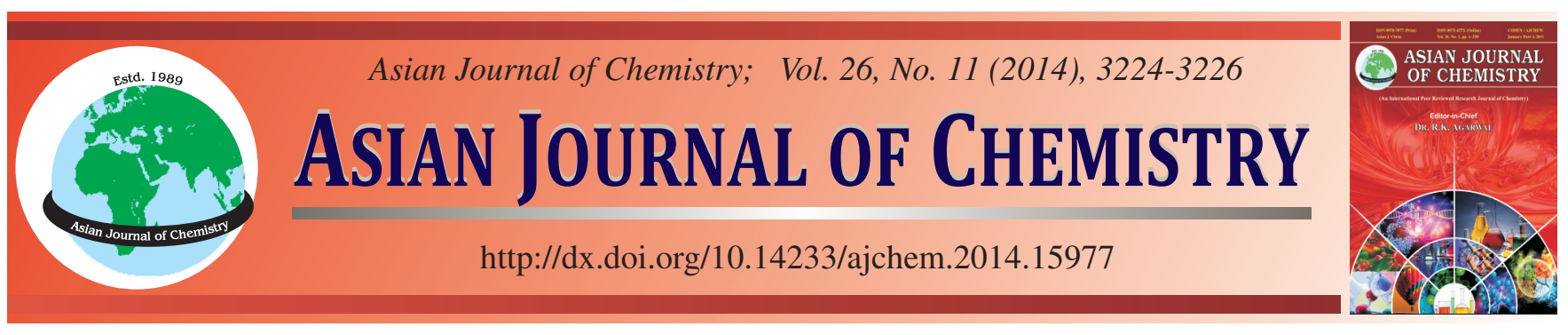

\title{
Synthesis and Characterization of Carbon Fiber Functionalized with Poly(glycidyl methacrylate) via Catalytic Chain Transfer Polymerization
}

\author{
Jingsong Sun, Lei Xiong ${ }^{*}$, Hongbo Liang ${ }^{*}$, Zhili Zuo, Hongring Gao, Longyu Luo and Wei Zhu
}

School of Material Science and Engineering, Nanchang Hangkong University, Nanchang 330063, P.R. China

*Corresponding authors: Tel: +86 791 83863025; E-mail: x_lei81@163.com; liahongbo@163.com

\begin{abstract}
Catalytic chain transfer polymerization (CCTP) is an extremely efficient process to produce low-molecular weight polymers. In this paper, we reported a controllable synthesis of carbon fibers functionalized with poly(glycidyl methacrylate) (CF-PGMA) by using cobalt oxime boron fluoride $(\mathrm{CoBF})$ as a chain transfer catalyst via catalytic chain transfer polymerization. In a typical run, the carbon fiber was first oxidized with a mixture of nitric acid and sulfuric acid (1:3) to introduce hydroxyl groups onto the carbon fiber surface. Subsequently, 3-(trimethoxysilyl)propylmethacrylate (KH570) was grafted onto the carbon fiber surface by chemical reaction of hydroxyl groups with the silane coupling agent. Finally, CF-PGMA was prepared by poly(glycidyl methacrylate) surface-grafted onto the carbon fiber via catalytic chain transfer polymerization and was characterized by FT-IR, TGA and XPS. The results show that the carbon fiber functionalized with poly(glycidyl methacrylate) was synthesized successfully and the content of grafted polymers was about $36.4 \%$.
\end{abstract}

Keywords: Carbon fiber, Catalytic chain transfer polymerization, Poly(glycidyl methacrylate), Surface modification.

\section{INTRODUCTION}

During the past decade, carbon fibers (CF) have been extensively studied for their high stiffness, high specific strength and chemical stability, which have been used in many different fields, such as aerospace industry, steamship industry, automotive industry, petroleum and chemical industry ${ }^{1-3}$. However, carbon fibers are difficult to form a strong interfacial adhesion between surface and resin matrix due to their smooth surface, inferior wettability and lower surface energy $y^{4-6}$. Therefore, many research works usually focus on how to modify the surface of carbon fiber and various attempts have been made, such as chemical method, plasma treatment, electrochemical method and surface functional groups grafting procedure ${ }^{7-13}$. Among these methods, grafting polymer on carbon fibers is an effective way to strengthen interfacial bond between carbon fiber surface and matrix.

In this paper, we mostly focused on the grafting poly(glycidyl methacrylate) (PGMA) chains from the carbon fiber surface via catalytic chain transfer polymerization (CCTP) for the purpose of improving and enhancing the fiber-matrix interfacial strength. The resulted CF-PGMA was characterized by FT-IR, TGA and XPS analyses.

\section{EXPERIMENTAL}

Fourier transform infrared (FT-IR) spectra $\left(400-4000 \mathrm{~cm}^{-1}\right)$ of the products were conducted on a Bruker 210 IR spectro- meter at room temperature. Each step of products was determined by thermal gravimetric analysis (TGA) on a Shimadzu TG-50. The samples were heated at a heating rate of $10{ }^{\circ} \mathrm{C} / \mathrm{min}$ from $25^{\circ} \mathrm{C}$ to $700{ }^{\circ} \mathrm{C}$ under nitrogen atmosphere. X-ray photoelectron spectroscopy (XPS) was performed on a VG ESCALAB MK II system with $\mathrm{MgK}_{\alpha}$ radiation.

The used carbon fibers were purchased from Hexcel. 3(Trimethoxysilyl)propylmethacrylate (KH570) were obtained from Arcos. Glycidyl methacrylate (GMA, Shanghai Reagents Co., Ltd) was washed by $5 \%$ sodium hydroxide solution and was then distilled under reduced pressure to remove the inhibitor before polymerization. 2,2'-Azobisisobutyronitrile (AIBN) (Aldrich) was recrystallized with methanol before use. Cobalt oxime boron fluoride $(\mathrm{CoBF})$ as cobalt catalyst was prepared based on the literature ${ }^{14}$.

Preparation of silane coupling agent functionalized carbon fiber: For the chemical oxidation of carbon fiber, the pristine carbon fibers were immersed in the solution of concentrated nitric acid and sulfuric acid in 1:3 ratio and the mix solution was up to $8 \mathrm{~h}$ in ultrasonic oscillation. Then the acidized fibers were diluted slowly and continuously with distilled water for $6 \mathrm{~h}$. The oxidized carbon fibers were then modified by silane coupling agent (KH570). At the end of reaction, the functionalized carbon fibers were vacuum filtered by using a $0.22 \mu \mathrm{m}$ poly(tetrafluoroethylene) (PTFE) membrane and the products were washed with anhydrous ethanol for three 
times to remove excess KH570. Finally, the silane coupling agent functionalized carbon fibers (CF- KH570) was dried under vacuum at $40{ }^{\circ} \mathrm{C}$ for $48 \mathrm{~h}$.

Surface-grafted PGMA of carbon fibers via CCTP: In a typical run, a mixture of CF-KH570, AIBN, CoBF, GMA was firstly dispersed in DMF in a flask. The flask was purged with nitrogen for 15 min to eliminate the oxygen, when the container was ensured to seal and under ultrasonic irradiation. Then the reaction mixture was stirred magnetically in oil bath maintaining constant reaction condition at $60^{\circ} \mathrm{C}$ for $20 \mathrm{~h}$. After reaching reaction time, the reaction was ended in ice bath and quickly added $10 \mathrm{~mL}$ of methanol. The resulted products were separated by centrifugation and washed with toluene/acetone mixture solvents for several times and dried under vacuum at $30{ }^{\circ} \mathrm{C}$ for $48 \mathrm{~h}$. The final products were marked as CF-PGMA. The procedure of PGMA grafted from carbon fiber surface is presented in Fig. 1.
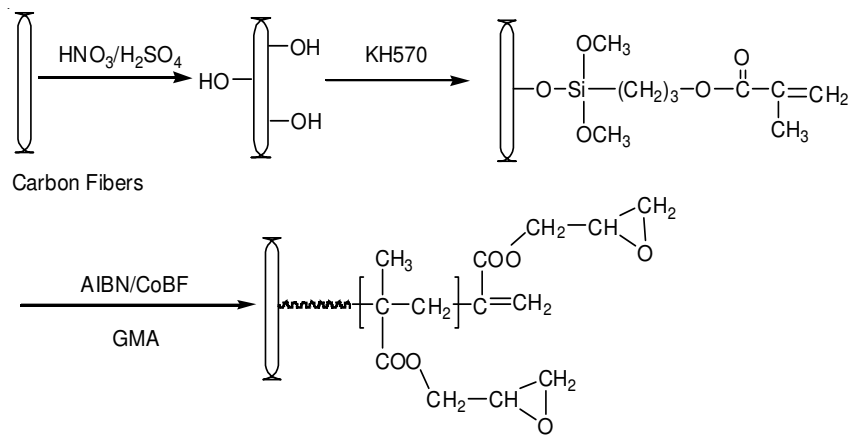

Fig. 1. Grafting of PGMA chains from carbon fibers

\section{RESULTS AND DISCUSSION}

As described, CoBF was demonstrated to catalyze the chain transfer to monomer reaction, which could produce a dead polymer chain with a terminal double bond and a monomeric radical. Therefore, we can take full advantage of this characteristic of CCTP to prepare CF-PGMA via the graft polymerization.

Characterization of silane coupling agent functionalized carbon fiber: Fig. 2 shows the FT-IR spectra of pristine carbon fiber and CF-KH570. The FT-IR spectrum of pristine carbon fiber (Fig. 2a) presents an obvious absorption peak around $3460 \mathrm{~cm}^{-1}$, due to the stretching vibration of $-\mathrm{OH}$ groups. Compared to the pristine carbon fiber, Fig. $2 b$ is the sample of CF-KH570, the new absorption peaks at $2925 \mathrm{~cm}^{-1}$ and $2850 \mathrm{~cm}^{-1}$ are assigned to $-\mathrm{CH}_{2}$ - groups of the grafted coupling agent. The peak at $1736 \mathrm{~cm}^{-1}$ is attributable to $\mathrm{C}=\mathrm{O}$ stretching vibrations, indicating that the coupling agent is immobilized onto the carbon fiber surface.

Characterization of the carbon fibers surface-grafted PGMA: The carbon fiber functionalized with PGMA was synthesized via CCTP process. The resulting products were referred to as CF-PGMA and characterized by FT-IR, TGA and XPS. Fig. 3 shows the FT-IR spectrum of CF-PGMA, the peaks at $2925 \mathrm{~cm}^{-1}$ and $2850 \mathrm{~cm}^{-1}$ belong to the aliphatic hydrogen $\left(-\mathrm{CH}_{2}\right)$ groups of GMA. A strong absorption peak around $1736 \mathrm{~cm}^{-1}$ is assigned to the ester carbonyl $(-\mathrm{C}=\mathrm{O})$ associated with GMA. In addition, there is a new peak at 910 $\mathrm{cm}^{-1}$ attributed to the stretching vibration of epoxy groups. It



Fig. 2. FT-IR spectra of (a) pristine carbon fiber and (b) CF-KH570



Fig. 3. FT-IR spectrum of CF-PGMA

is clearly found that CF-PGMA was successfully grafted onto the carbon fiber surface via CCTP.

Thermogravimetric measurements were performed on the pristine carbon fiber, CF-KH570 and CF-PGMA to determine the amount of grafted polymer on the carbon fiber surface under a nitrogen atmosphere. The TGA results are shown in the Fig. 4. In order to achieve exact results of TGA, all the samples were isolated and dried under vacuum for several times. As shown in Fig. 4a, the pristine carbon fiber decomposes slowly with the increasing of temperature between $25^{\circ} \mathrm{C}$ and $700{ }^{\circ} \mathrm{C}$ and the weight loss is about $4.8 \%$. This may be assigned to the weight loss of hydroxyl groups or absorbed water on the carbon fiber surface. A weight loss of $11.6 \%$ was observed for the CF-KH570 (Fig. 4b). This weight loss results from the losing of silane coupling agent grafted onto the surface of carbon fiber. Compared with the pristine carbon fiber and CF-KH570, the thermal curve of CF-PGMA (Fig. $4 c)$ shows a major decomposition at a temperature range between $300{ }^{\circ} \mathrm{C}$ and $470{ }^{\circ} \mathrm{C}$ corresponding to PGMA grafting on the carbon fiber surface. The amount of grafted polymer calculated from TGA data is about $36.4 \%$. 


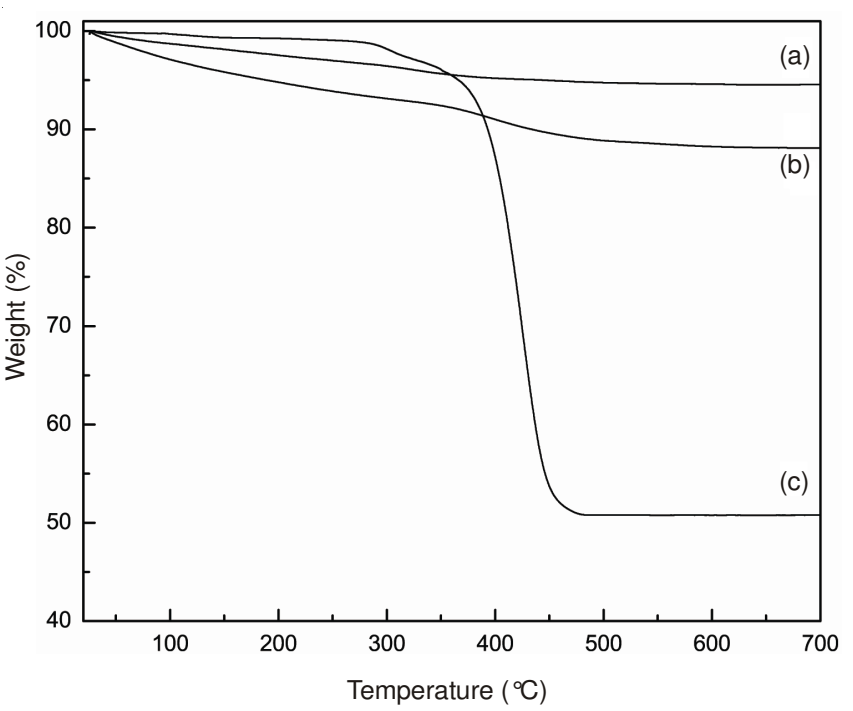

Fig. 4. TGA curves of (a) pristine carbon fiber, (b) CF-KH570 and (c) CFPGMA

$\mathrm{X}$-ray photoelectron spectroscopic analysis was used to further characterize CF-KH570 and CF-PGMA and the results are shown in Fig. 5. From Fig. 5a, for CF-KH570, the major peak component at the binding energy (BE) of $532.6 \mathrm{eV}$ and the minor peak component at the $\mathrm{BE}$ of $285.4 \mathrm{eV}$ are assigned to $\mathrm{O} 1 \mathrm{~s}$ and $\mathrm{C} 1 \mathrm{~s}$, respectively. Meanwhile, peaks at the binding energy of $150.8 \mathrm{eV}$ and $101.9 \mathrm{eV}$ corresponded to $\mathrm{Si} 2 \mathrm{~s}$ and $\mathrm{Si} 2 \mathrm{p}$ are also found in this spectrum, indicating that the coupling agent was grafted on the carbon fiber surface. Compared with the CF-KH570, we can find in the Fig. 5b that the peaks for $\mathrm{Si} 2 \mathrm{~s}$ at $150.8 \mathrm{eV}$ and $\mathrm{Si} 2 \mathrm{p}$ at $101.9 \mathrm{eV}$ turn very weak. On the contrary, the peak for $\mathrm{C} 1 \mathrm{~s}$ at binding energy of $285.4 \mathrm{eV}$ grows

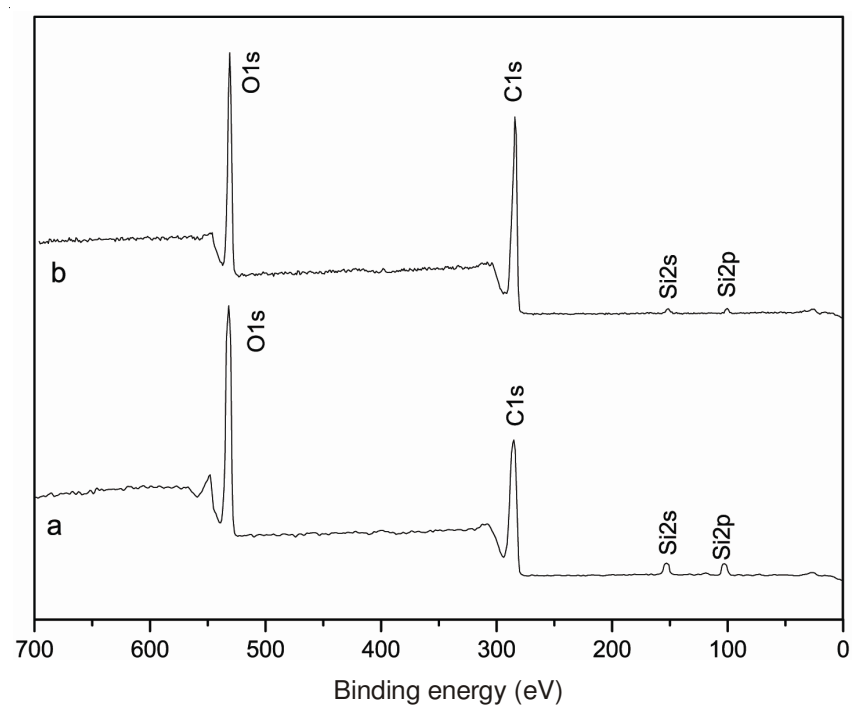

Fig. 5. XPS spectra of (a) CF-KH570 and (b) CF-PGMA strong, shows that the polymer chains are grafted onto the carbon fiber surface. As a result, the FT-IR, TGA and XPS results confirm qualitatively that the PGMA was successfully grafted onto the carbon fiber surface via CCTP.

\section{Conclusion}

In this work, a new available strategy for prepared carbon fiber surface-grafted PGMA via CCTP technique had been successfully realized. Firstly, we adopted KH570 as the silane coupling agent to prepare vinyl-functionalized carbon fiber. Subsequently, the introduced double bonds on the surface of functionalized carbon fiber allowed them to further graft with GMA via CCTP in situ. Finally, controllable synthesis of welldefined carbon fiber surface-grafted PGMA with terminal unsaturated double bond had been produced via CCTP process. The resulted CF-PGMA was characterized by means of FTIR, TGA and XPS. The results showed that the PGMA was successfully grafted from the carbon fiber surface. Furthermore, TGA analysis demonstrated that the content of the polymer grafted on the carbon fiber was about $36.4 \%$. A promising way to fabricate functionalized carbon fiber via CCTP technique would open up a new avenue for the preparation and application of carbon fiber-reinforced composites.

\section{ACKNOWLEDGEMENTS}

The authors are grateful for the financial support by National Natural Science Foundation of China (No. 51103069), Science Foundation of Aeronautics of China (No. 2011ZF56018) and Natural Science Foundation of Jiangxi Province (No. 20122BAB216014).

\section{REFERENCES}

1. S.J. Park, M.K. Seo and Y.S. Lee, Carbon, 41, 723 (2003).

2. H. Ogawa and K. Saito, Carbon, 33, 783 (1995).

3. Z.H. Wu, C.U. Pittman Jr. and S.D. Gardner, Carbon, 33, 597 (1995).

4. C. Marieta, E. Schulz, L. Irusta, N. Gabilondo, A. Tercjak and I. Mondragon, Compos. Sci. Technol., 65, 2189 (2005).

5. S. Yumitori and Y. Nakanishi, Composites Part A, 27, 1059 (1996).

6. M. Toyoda, H. Katoh and M. Inagaki, Carbon, 39, 2231 (2001).

7. C.U. Pittman Jr., W. Jiang, Z.R. Yue, S. Gardner, L. Wang, H. Toghiani and C.A. Leon, Carbon, 37, 1797 (1999).

8. G.M. Wu, Mater. Chem. Phys., 85, 81 (2004).

9. Y.V. Basova, H. Hatori, Y. Yamada and K. Miyashita, Electrochem. Commun., 1, 540 (1999).

10. E. Theodoridou, A.D. Jannakoudakis, P.D. Jannakoudakis, P. Andonoglou and J.O. Besenhard, Synth. Met., 87, 225 (1997).

11. M.B. Ivanov, N.V. Gavrilov, T.A. Belyh, E.A. Ligacheva, L.V. Galijeva, A.E. Ligachev and V.V. Sohoreva, Surf. Coat. Technol., 201, 8326 (2007).

12. N. Dilsiz, N.K. Erinc, E. Bayramli and G. Akovali, Carbon, 33, 853 (1995).

13. L. Xiong, X.F. Li, H.B. Liang and S.M. Huang, Asian J. Chem, 25, 4032 (2013).

14. A. Bakac and J.H. Espenson, J. Am. Chem. Soc., 106, 5197 (1984). 